\title{
Blind PARAFAC Signal Detection for Polarization Sensitive Array
}

\author{
Xiaofei Zhang and Dazhuan Xu \\ Electronic Engineering Department, Nanjing University of Aeronautics and Astronautics, Nanjing 210016, China
}

Received 27 September 2006; Revised 22 January 2007; Accepted 16 April 2007

Recommended by Nicola Mastronardi

This paper links the polarization-sensitive-array signal detection problem to the parallel factor (PARAFAC) model, which is an analysis tool rooted in psychometrics and chemometrics. Exploiting this link, it derives a deterministic PARAFAC signal detection algorithm. The proposed PARAFAC signal detection algorithm fully utilizes the polarization, spatial and temporal diversities, and supports small sample sizes. The PARAFAC algorithm does not require direction-of-arrival (DOA) information and polarization information, so it has blind and robust characteristics. The simulation results reveal that the performance of blind PARAFAC signal detection algorithm for polarization sensitive array is close to nonblind MMSE method, and this algorithm works well in array error condition.

Copyright (c) 2007 X. Zhang and D. Xu. This is an open access article distributed under the Creative Commons Attribution License, which permits unrestricted use, distribution, and reproduction in any medium, provided the original work is properly cited.

\section{INTRODUCTION}

Polarization sensitive arrays have some inherent advantages over traditional antenna arrays, since they have the capability of separating signals based on their polarization characteristics, as well as spatial diversity. Intuitively, polarization sensitive antenna arrays will provide significant improvements for signals which have different polarization characteristics. Polarization sensitive arrays are used widely in the communication, radio and navigation $[1,2]$. Maximum likelihood signal estimation method for polarization sensitive arrays is proposed in [3]. Filtering performance of polarization sensitive array in completely polarized case is investigated in [4]. The methods mentioned above are nonblind methods, since they require the knowledge of DOA and polarization information. Blind parallel factor (PARAFAC) signal detection algorithm for polarization sensitive array is investigated in this paper.

PARAFAC analysis has been first introduced as a data analysis tool in psychometrics, most of the research in the area is conducted in the context of chemometrics [5], spectrophotometric, chromatographic, and flow injection analyses. Harshman [6] developed the PARAFAC model. At the same time, Caroll and Chang [7] introduced the canonical decomposition model, which is essentially identical to PARAFAC. In signal processing field, PARAFAC can be thought of as a generalization of ESPRIT and joint approximate diagonalization ideas $[8,9]$. PARAFAC is thus naturally related to linear algebra for multiway arrays [10]. PARAFAC is used widely in blind receiver detection for direct-sequence code-division multiple-access (CDMA) system [11], array signal processing $[12,13]$, blind estimation of multi-input multi-output (MIMO) system [14], blind speech separation [15], downlink receiver for space-time block-boded CDMA system [16], and multiuser detection for single-input multioutput (SIMO) CDMA System [17].

Our work links the polarization-sensitive-array signal detection problem to the parallel factor model and derives a deterministic blind PARAFAC signal detection whose performance is close to nonblind minimum mean-squared error (MMSE). The proposed PARAFAC supports small sample sizes, and even works well in array error condition. Most notably, it does not require knowledge of the DOA and polarization information. Instead, PARAFAC relies on a fundamental result of Kruskal [10] regarding the uniqueness of low-rank three-way array decomposition.

This paper is structured as follows: Section 2 develops data model, Section 3 discusses identifiability issues and deals with algorithmic issues, Section 4 presents simulation results, and Section 5 summarizes our conclusions.

\section{THE RECEIVED SIGNAL MODEL FOR POLARIZATION SENSITIVE ARRAY}

Crossed dipoles are shown in Figure 1. Each dipole in the array is a short dipole, so the output voltage from each dipole 


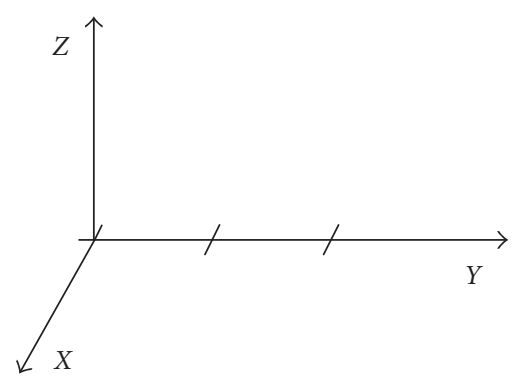

FIGURE 1: The structure of polarization sensitive array.

is proportional to the electric field component along that dipole. There are orthogonal short dipoles, the $x$ - and $y$-axis dipoles, parallel to the $x$ - and $y$-axes, respectively. The $m$ th dipole pair, $m=1,2, \ldots, M$, has its center on the $y$-axis at $y=(m-1) d$. The distance $d$ between two adjacent dipole pairs is assumed to be a half-wavelength to avoid angle ambiguity problems. We consider signals in the far-field, in which case the signal sources are far enough away that the arriving waves are essentially planes over the length of the array. Assume that the noise is independent of the source, and noise is additive i.i.d. Gaussian.

\subsection{The received signal model for polarization sensitive antenna}

We begin by considering the polarization of an incoming signal. Supposing that an antenna is at the origin of a spherical coordinate system, and a signal $b(t)$ is arriving from direction $\theta, \varphi$, where $\varphi$ is the elevation angle and $\theta$ is the azimuth angle. Let this signal be a transverse electromagnetic (TEM) wave, and consider the polarization ellipse produced by the electric field in a fixed transverse plane. Polarization parameters are $\gamma, \eta$. We characterize the antenna in terms of its response to linearly polarized signals in the $x$ and $y$ directions. Let $v_{x}$ be the complex voltage induced at the antenna output terminals by an incoming electromagnetic signal with a unit electric field polarized entirely in the $x$ direction. Similarly, let $v_{y}$ be the output voltages induced by signals with unit electric fields polarized in the $y$ directions. According to [4], the total output voltage from polarization antenna is

$$
y_{p}(t)=\left[\begin{array}{cc}
\cos \theta \cos \varphi & -\sin \varphi \\
\cos \theta \sin \varphi & \cos \varphi
\end{array}\right]\left[\begin{array}{c}
\sin \gamma e^{j \eta} \\
\cos \gamma
\end{array}\right] b(t)=s b(t),
$$

where

$$
s=\left[\begin{array}{cc}
\cos \theta \cos \varphi & -\sin \varphi \\
\cos \theta \sin \varphi & \cos \varphi
\end{array}\right]\left[\begin{array}{c}
\sin \gamma e^{j \eta} \\
\cos \gamma
\end{array}\right]
$$

is the polarization vector, and it relates to polarization and DOA information.

\subsection{The received signal model for polarization sensitive array}

Assume that a signal $b(t)$ arrives at the uniform linear array with $M$ pairs of crossed dipoles. The received signal of the polarization sensitive array is shown as follows:

$$
y(t)=\left[s^{T}, q s^{T}, \ldots, q^{M-1} s^{T}\right]^{T} b(t)=(a \otimes s) b(t),
$$

where $\otimes$ is Kronecker product, $s$ is the polarization vector, $a=\left[1, q, \ldots, q^{M-1}\right]^{T}$ is the direction vector, $q=e^{-j 2 \pi d \sin \theta / \lambda}$.

When $\mathbf{K}$ sources impinge the polarization sensitive array, the received signal at the output of the polarization sensitive array is

$$
x=\left[a_{1} \otimes s_{1}, a_{2} \otimes s_{2}, \ldots, a_{K} \otimes s_{K}\right] B^{T},
$$

where $a_{i}$ and $s_{i}$ are the direction vector and polarization vector of the $i$ th source, respectively, and $B=\left[b_{1}^{T}, b_{2}^{T}, \ldots, b_{K}^{T}\right]$ is the source matrix with $N \times K$, where $b_{i}$ is the transmit signal of the $i$ th source.

Equation (4) can be denoted as

$$
x=[A \circ S] B^{T},
$$

where $A \circ S$ is Khatri-Rao product, $A=\left[a_{1}, a_{2}, \ldots, a_{K}\right]$ is the direction matrix, and $S=\left[s_{1}, s_{2}, \ldots, s_{K}\right]$ is the polarization matrix.

Equation (5) can be denoted as

$$
x=\left[\begin{array}{c}
X . .1 \\
X . .2 \\
\vdots \\
X . . M
\end{array}\right]=\left[\begin{array}{c}
S D_{1}(A) \\
S D_{2}(A) \\
\vdots \\
S D_{M}(A)
\end{array}\right] B^{T},
$$

where $D_{m}(\cdot)$ is understood as an operator that extracts the $m$ th row of its matrix argument and constructs a diagonal matrix out of it, $D_{m}(A)=\operatorname{diag}\left(\left[a_{m, 1}, a_{m, 2}, \ldots, a_{m, K}\right]\right)$.

Use slices to denote

$$
X . . m=S D_{m}(A) B^{T}, \quad m=1,2, \ldots, M,
$$

where $X . . m$ is the $m$ th slice in spatial direction.

In the presence of noise, the received signal model becomes

$$
\tilde{X}_{\cdot \cdot m}=X{ }_{\cdot \cdot m}+V \cdot \cdot m=S D_{m}(A) B^{T}+V \cdot \cdot m, \quad m=1,2, \ldots, M,
$$

where $V . . m$, the $2 \times N$ matrix, is the received noise corresponding to the $m$ th slice.

The signal in (7) is also denoted through rearrangements as

$$
\begin{aligned}
x_{m, n, p}=\sum_{f=1}^{K} a_{m, f} s_{n, f} b_{p, f}, \quad m & =1, \ldots, M ; \\
n & =1, \ldots, N ; p=1,2,
\end{aligned}
$$


where $a_{m, f}$ stands for the $(m, f)$ element of $A$ matrix, and similarly for the others. Note that (9) is a sum of triple products; it is well known as the trilinear model, trilinear decomposition, canonical decomposition, or PARAFAC analysis. The trilinear model $\mathbf{X}$ reflects three different kinds of diversities available: spatial, temporal, and polarization diversities. Another view, $X ._{m}=S D_{m}(A) B^{T}, m=1,2, \ldots, M$, can be interpreted as slicing the $3 \mathrm{D}$ data in a series of slices $(2 \mathrm{D}$ data) along the spatial direction. The symmetry of the trilinear model in (9) allows two more matrix system rearrangements, which can be interpreted as slicing the three-way data along different directions. In particular,

$$
Y . \cdot p=B D_{p}(S) A^{T}, \quad p=1,2,
$$

where the $N \times M$ matrix $Y_{. . p}=\left[x_{., \cdot, p}\right] . Y_{. . p}$ is the $p$ th slice in polarization direction. Similarly,

$$
Z \cdot . n=A D_{n}(B) S^{T}, \quad n=1,2, \ldots, N
$$

where the $M \times 2$ matrix $Z_{._{n}}=\left[x_{n, \cdot, \cdot] .} Z_{._{n}}\right.$ is the $n$th slice in the temporal direction.

\section{BLIND PARAFAC SIGNAL DETECTION FOR POLARIZATION SENSITIVE ARRAY}

\subsection{Trilinear alternating least squares}

Trilinear alternating least square (TALS) algorithm is the common data detection method for trilinear model [6]. The basic idea of TALS is as follows: (a) each time, update a matrix using least squares conditioned on previously obtained estimates of the remaining matrix; (b) proceed to update another matrix; (c) repeat until convergence. TALS algorithm is discussed in detail as follows.

According to (6), least squares fitting is

$$
\min _{A, S, B}\left\|\left[\begin{array}{c}
\tilde{X} \cdot \cdot 1 \\
\tilde{X} \cdot \cdot 2 \\
\vdots \\
\tilde{X} \cdot \cdot M
\end{array}\right]-\left[\begin{array}{c}
S D_{1}(A) \\
S D_{2}(A) \\
\vdots \\
S D_{M}(A)
\end{array}\right] B^{T}\right\|_{F},
$$

where $\|\cdot\|_{F}$ stands for the Frobenius norm. $\tilde{X}_{. . m}, m=$ $1,2, \ldots, M$, are the noisy slices.

Least squares update for $B$ is

$$
\widehat{B}^{T}=\left[\begin{array}{c}
\hat{S} D_{1}(\hat{A}) \\
\hat{S} D_{2}(\hat{A}) \\
\vdots \\
\widehat{S} D_{M}(\hat{A})
\end{array}\right]^{+}\left[\begin{array}{c}
\tilde{X}_{. \cdot 1} \\
\tilde{X} \cdot .2 \\
\vdots \\
\tilde{X} \cdot{ }_{\cdot M}
\end{array}\right]
$$

where $[\cdot]^{+}$stands for pseudoinverse. $\hat{A}$ and $\hat{S}$ denote previously obtained estimates of $A$ and $S$.

Similarly, from the second way of slices, $Y . . p=$ $B D_{p}(S) A^{T}, p=1,2$, which is rewritten as

$$
\left[\begin{array}{l}
Y . \cdot 1 \\
Y \cdot{ }_{2}
\end{array}\right]=\left[\begin{array}{l}
B D_{1}(S) \\
B D_{2}(S)
\end{array}\right] A^{T},
$$

LS fitting is

$$
\min _{A, S, B}\left\|\left[\begin{array}{c}
\tilde{Y}_{\cdot .1} \\
\tilde{Y}_{\cdot .2}
\end{array}\right]-\left[\begin{array}{l}
B D_{1}(S) \\
B D_{2}(S)
\end{array}\right] A^{T}\right\|_{F},
$$

and the LS update for $A$ is

$$
\widehat{A}^{T}=\left[\begin{array}{l}
\hat{B} D_{1}(\hat{S}) \\
\hat{B} D_{2}(\widehat{S})
\end{array}\right]^{+}\left[\begin{array}{l}
\tilde{Y}_{\cdot \cdot 1} \\
\tilde{Y}_{\cdot \cdot 2}
\end{array}\right] .
$$

Finally, from the third way of slices, $Z . \cdot n=A D_{n}(B) S^{T}, n=$ $1,2, \ldots, N$. And then LS update for $S$ is

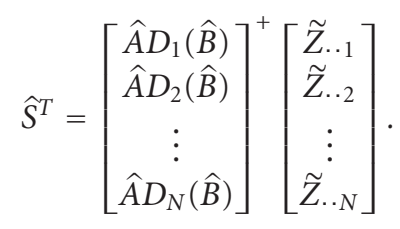

The loss function to be minimized is the sum of squared residuals (SSR) in the TALS algorithm:

$$
\mathrm{SSR}=\sum_{m=1}^{M} \sum_{n=1}^{N} \sum_{p=1}^{2} e_{m, n, p}^{2}
$$

where $e_{m, n, p}=\tilde{x}_{m, n, p}-\sum_{f=1}^{K} \hat{a}_{m, f} \hat{s}_{n, f} \hat{b}_{p, f}$ is the $(m, n, p)$ element of fitting error. $\hat{a}_{m, f}$ stands for the $(m, f)$ element of $\hat{A}$, and similarly for the others.

According to (13), (16), and (17), matrices $B, A$, and $S$ are updated with conditioned least squares, respectively. The matrix update will stop until convergence.

TALS is optimal when noise is additive i.i.d. Gaussian [18]. TALS algorithm has several advantages: it is easy to implement, guarantee to converge, and simple to extend to higher order data. TALS algorithm is known to suffer from degeneracy and slow convergence. Although a unique solution exists, it is not always guaranteed to be found as the TALS algorithm can be stuck in local minima [19]. TALS can be initialized by eigen-decomposition to speed up convergence [12]. According to (10), the two slices along the polarization direction are represented as

$$
Y_{{ }_{1} 1}=B A_{E}, \quad Y_{\cdot 2}=B D A_{E}
$$

where $A_{E}=D_{1}(S) A^{T}$ and $D=D_{2}(S) D_{1}(S)^{-1}$.

Construct auto- and cross-correlation matrices:

$$
\begin{gathered}
R_{1}=Y_{\cdot \cdot 1}^{H} Y . \cdot 1=A_{E}^{H} B^{H} B A_{E}, \\
R_{2}=Y_{\cdot \cdot 1}^{H} Y . \cdot 2=A_{E}^{H} B^{H} B D A_{E} .
\end{gathered}
$$

Define $\alpha=A_{E}^{H} B^{H} B$, then

$$
R_{1}=\alpha A_{E}, \quad R_{2}=\alpha D A_{E} .
$$

According to (21),

$$
\alpha^{+} R_{1}=D^{-1} \alpha^{+} R_{2},
$$


where $[\cdot]^{+}$is the pseudoinverse. Let $u_{f}^{H}$ be the $f$ th row of $\alpha^{+}$ and let $\lambda_{f}$ be the $f$ th element along the diagonal of $D^{-1}$. The general eigen-decomposition for $\left(R_{1}, R_{2}\right)$ is given as

$$
u_{f}^{H}\left(R_{1}-\lambda_{f} R_{2}\right)=0, \quad f=1,2, \ldots, K .
$$

The $\lambda_{f}$ 's and $u_{f}^{H}$ 's are the generalized eigenvalues and left generalized eigenvectors of $\left(R_{1}, R_{2}\right)$. Once $\alpha^{+}$is recovered, then $A_{E}=\alpha^{+} R_{1}, B=Y_{. .1}\left[A_{E}\right]^{+}$, and $D=B^{+} Y_{. .2}\left[A_{E}\right]^{+}$.

\subsection{Identifiablity}

The $k$-rank concept is very important in the trilinear algebra.

Definition 1 (see [10]). Consider a matrix $A \in C^{I \times J}$. If $\operatorname{rank}(A)=r$, then $A$ contains a collection of $r$ linearly independent columns. Moreover, if every $l \leq J$ columns of $A$ are linearly independent, but this does not hold for every $l+1$ columns, then $A$ has $k$-rank $k_{A}=l$. Note that $k_{A} \leq \operatorname{rank}(A)$, for all $A$.

Theorem 1 (see [20]). $X . . m=S D_{m}(A) B^{T}, m=1,2, \ldots, M$, where $A \in C^{M \times K}, S \in C^{2 \times K}$, and $B \in C^{N \times K}$, considering that $\mathrm{A}$ is a matrix with Vandermonde characteristic. If

$$
k_{S}+\min \left(M+k_{B}, 2 K\right) \geq 2 K+2,
$$

then $A, B$, and $S$ are unique up to permutation and scaling of columns, that is to say, any other triple $\bar{A}, \bar{B}, \bar{S}$ that constructs $X . . m(m=1,2, \ldots, M)$ is related to $A, B$, and $S$ via

$$
\bar{A}=A \Pi \Delta_{1}, \quad \bar{B}=B \Pi \Delta_{2}, \quad \bar{S}=S \Pi \Delta_{3},
$$

where $\Pi$ is a permutation matrix, and $\Delta_{1}, \Delta_{2}$, and $\Delta_{3}$ are diagonal scaling matrices satisfying

$$
\Delta_{1} \Delta_{2} \Delta_{3}=I \text {. }
$$

Scale ambiguity and permutation ambiguity are inherent to the separation problem. This is not a major concern. Permutation ambiguity can be resolved by resorting to a priori or embedded information. The scale ambiguity can be resolved using automatic gain control and differential encoding/decoding [21] or embedded information.

Although the PARAFAC uniqueness result is purely deterministic, it also admits statistical characteristics. A matrix whose columns are drawn independently from an absolutely continuous distribution has full rank with probability one, even when the elements across a given column are dependent random variables [11]. In our present context, for source-wise independent source signals, $k_{B}=\min (N, K)$; for source-wise independent polarization, $k_{S}=\min (2, K)$, and therefore, (24) becomes

$$
\min (2, K)+\min (M+\min (N, K), 2 K) \geq 2 K+2 .
$$

In practice, $K \geq 2, \min (2, K)=2$, hence the practical condition is

$$
M+\min (N, K) \geq 2 K .
$$

If $N \leq K$, the identifiable condition is $M+N \geq 2 K$.

If $N \geq K$, the identifiable condition is $M \geq K$, and then $\min (M, N)$ sources can be recovered.

If matrix A in Theorem 1 is not a Vandermonde matrix, according to [11], the identifiable condition is

$$
\min (2, K)+\min (N, K)+\min (M, K) \geq 2 K+2 .
$$

In practice, $K \geq 2$, then the identifiable condition is

$$
N \geq K, \quad M \geq K,
$$

so $\min (M, N)$ sources can be recovered $[8,22,23]$.

\section{SIMULATION RESULTS}

If $\mathbf{X}$ is the received signal without noise and $\hat{X}=X+V$ is the received noisy signal, we define the sample SNR as

$$
\mathrm{SNR}=10 \log _{10} \frac{\|X\|_{F}^{2}}{\|V\|_{F}^{2}} \mathrm{~dB},
$$

where $\|X\|_{F}^{2}$ is the sum of squares of all elements of the 3D data $X$.

As shown in Theorem 1, the scaling ambiguity and the permutation ambiguity are inherent to this blind separation problem. We remove the scaling ambiguity among the estimated source matrix via embedded information. Permutation ambiguity is resolved using a greedy least square matching algorithm [11].

A uniform linear array with 16 pairs of crossed dipoles is used in the simulation. Assume that each source only has one path to polarization sensitive array. We assume binary phase-shift keying (BPSK) modulated signal and additive gauss white noise. For all the simulation, the number of the sources is 3 . Note that $N$ is the number of snapshots.

We present Monte Carlo simulations that are to assess the bit error rate (BER) performance of the proposed blind PARAFAC signal detection algorithm. The number of Monte Carlo trials is 1000. The PARAFAC algorithm does not require DOA information and polarization information. We compare our PARAFAC algorithm with the nonblind MMSE receiver. MMSE receiver offers a performance bound against which blind algorithms are often measured [24, 25]. For the received signal in (5), the nonblind MMSE solution is

$$
\widehat{B}_{\text {MMSE }}^{T}=\left(\Lambda \Lambda^{H}+\frac{1}{\mathrm{SNR}}\right)^{-1} \Lambda^{H} \tilde{x}, \quad \text { where } \Lambda=A \circ S .
$$

Compared with our blind PARAFAC receiver, the nonblind MMSE receiver assumes the perfect knowledge of DOA, SNR, and polarization information.

The performances of these algorithms under different $N$ are shown in Figures 2-7.

Figures 2 and 3 present large sample results for $N=800$ and $N=400$, respectively. From Figures 2 and 3, we find that blind PARAFAC signal detection algorithm is very close to nonblind MMSE method. 


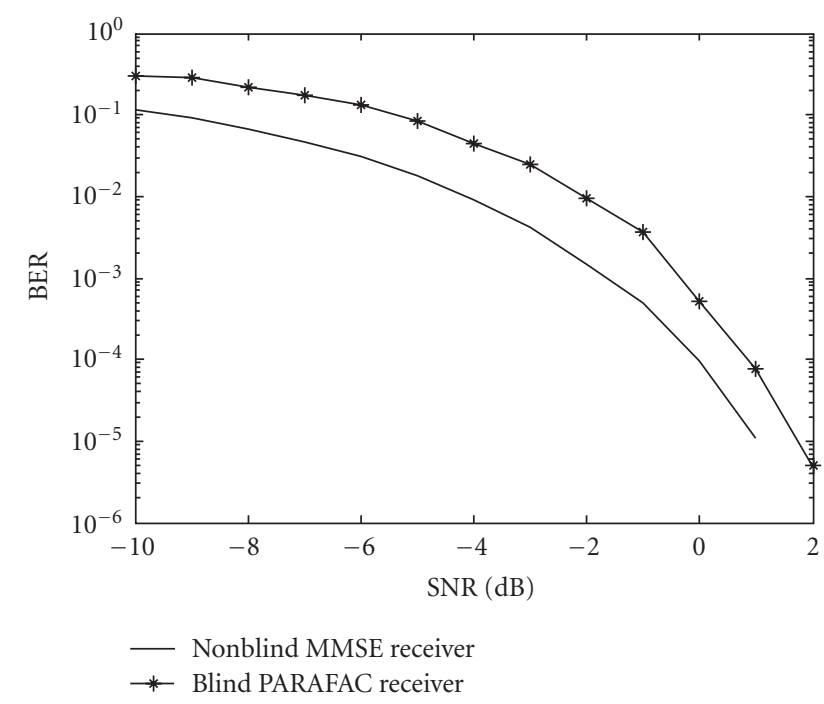

Figure 2: The algorithm performances comparison with $N=800$.

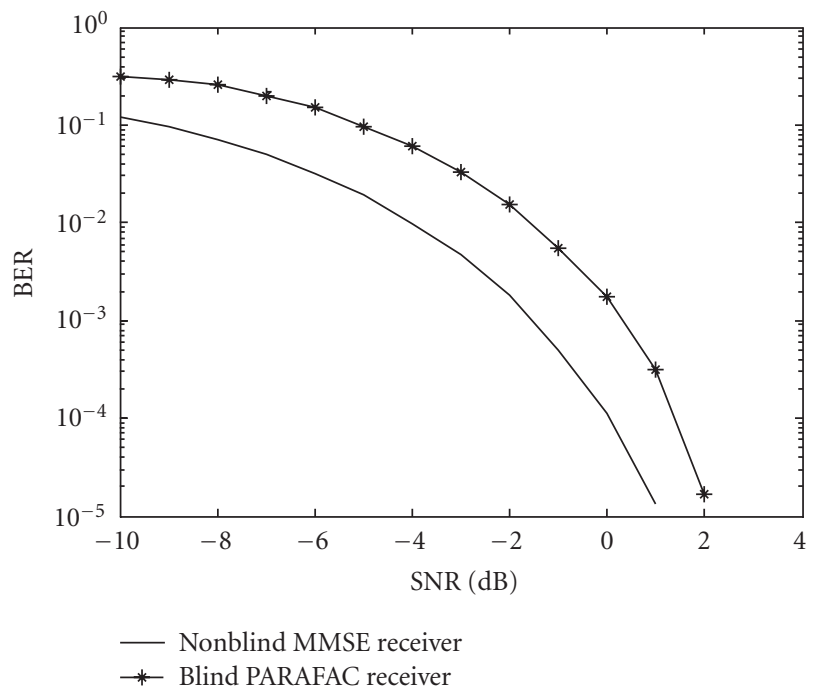

FIGURE 3: The algorithm performances comparison with $N=400$.

Figures 4 and 5 depict results for $N=200$ and 100, respectively. From Figures 2 to 5, we find that the gap between blind PARAFAC and (nonblind) MMSE increases as $N$ decreases.

Figures 6 and 7 show small sample results for $N=50$ and $N=20$, respectively. It is clear that PARAFAC performs well even for very small sample sizes.

The actual array parameters may differ from the nominal array in several ways: gain, phase, and sensor location errors. Gain and phase errors occur when the response of each antenna to a known signal has a different amplitude and/or phase response than expected. Blind PARAFAC signal detection algorithm performance in the array error condition is also investigated. In this simulation, array error vector is the

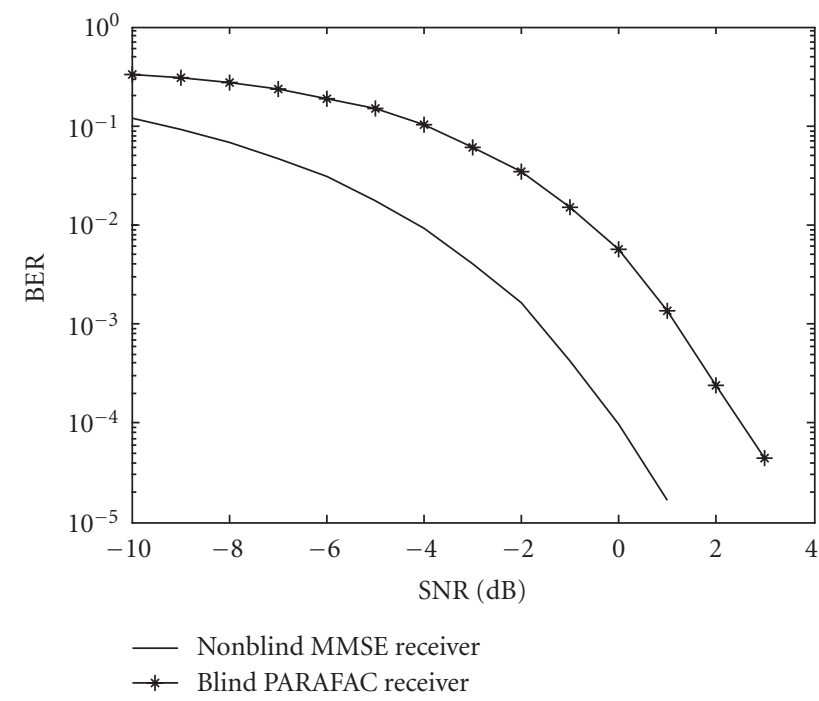

FIgURE 4: The algorithm performances comparison with $N=200$.

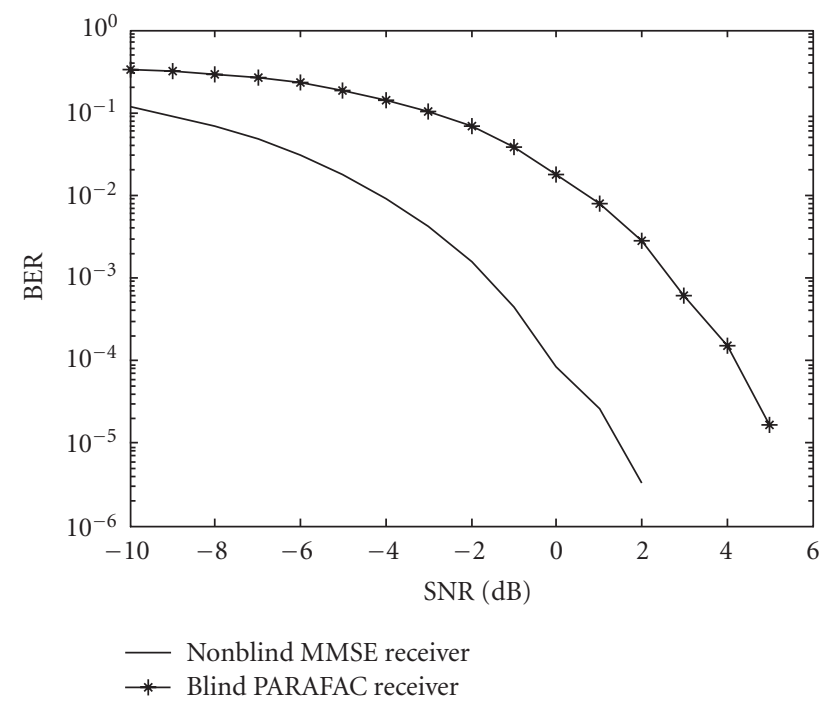

FIGURE 5: The algorithm performances comparison with $N=100$.

array gain and phase error vector. The array error vector $g=$ $[0.8523+0.3031,0.6071-0.4953 i, 0.7083+0.7059 i, 0.7497-$ $0.7167 i, 0.6931+0.8916 i, 0.8343+0.6883 i, 0.730+$ $0.6894 i, 0.6678-0.5133 i, 0.4806-0.9112 i, 0.6669+$ $0.5634 i, 0.7834-0.6828 i, 0.7497-0.7237 i, 0.6563+$ $0.82316 i, 0.8123+0.6823 i, 0.7245+0.6239 i, 0.6234-0.5133 i]$. Assume that array response vector for DOA $=\theta$ is $a(\theta)$, then the array response vector with array error is $\operatorname{diag}(g) a(\theta)$. The direction matrix with array error is not Vandermonde matrix, and then the identifiable condition is shown in (30). The sample number $N$ is 100 in this simulation. The performance of blind PARAFAC signal detection algorithm in array error condition is shown in Figure 8. Figure 8 shows that blind PARAFAC signal detection algorithm has the better 


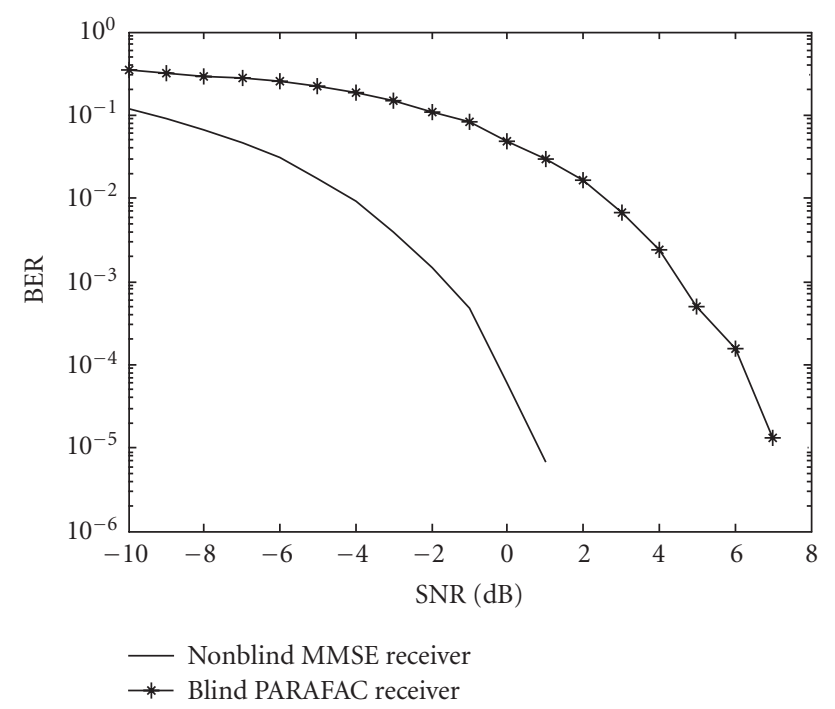

FIGURE 6: The algorithm performances comparison with $N=50$.

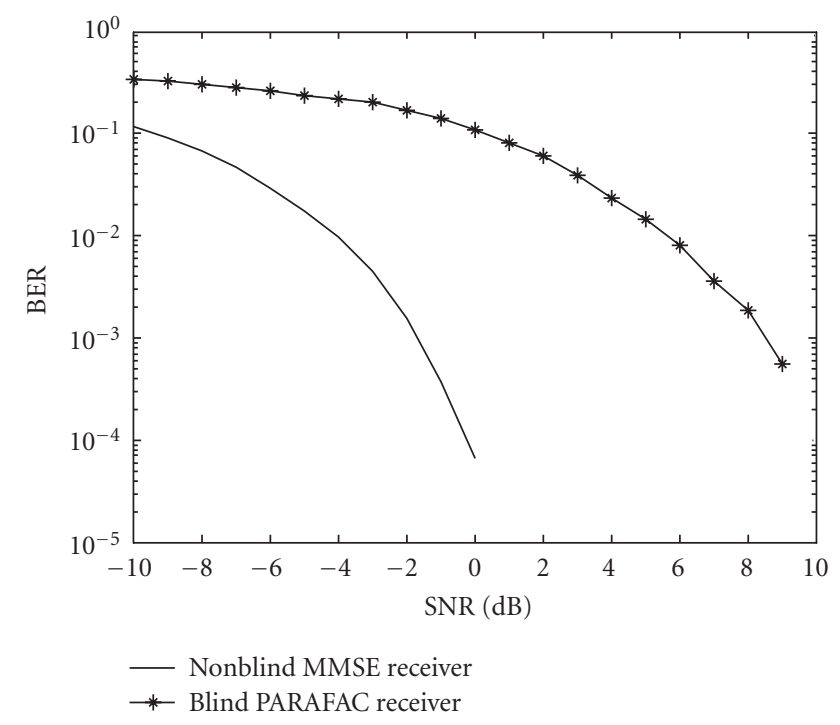

FIGURE 7: The algorithm performances comparison with $N=20$.

performance in the array error condition. Blind PARAFAC signal detection algorithm has robust characteristics to array error.

\section{CONCLUSIONS}

This paper has developed a link between PARAFAC analysis and blind signal detection for polarization sensitive array. Relying on the uniqueness of low-rank three-way array decomposition and trilinear alternating least squares, a deterministic PARAFAC signal detection algorithm has been proposed. The algorithm does not require DOA information and polarization information, and it has blind and robust characteristics. The simulation results reveal that the performance

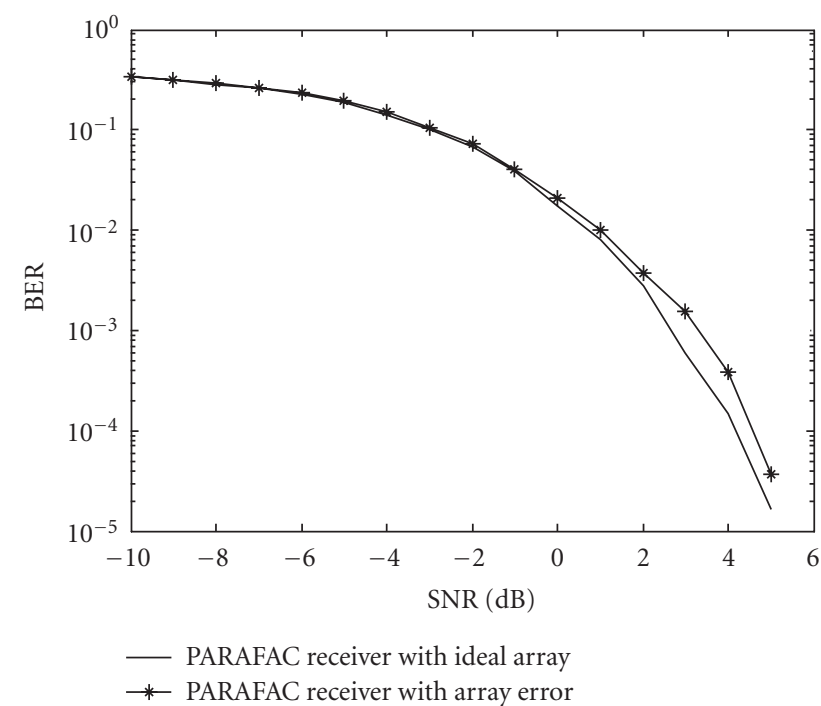

FIGURE 8: The algorithm performance with array error.

of blind PARAFAC signal detection algorithm for polarization sensitive array is close to nonblind MMSE method, and this algorithm works well in array error condition and supports small sample sizes.

\section{ACKNOWLEDGMENTS}

This work is supported by the startup fund of Nanjing University of Aeronautics and Astronautics (S0583-041) and Jiangsu NSF Grant BK2003089. The authors wish to thank the anonymous reviewers for valuable suggestions on improving this paper.

\section{REFERENCES}

[1] J. W. P. Ng and A. Monikas, "Polarisation-sensitive array in blind MIMO CDMA system," Electronics Letters, vol. 41, no. 17, pp. 970-972, 2005.

[2] I. Kaptsis and K. G. Balmain, "Base station polarizationsensitive adaptive antenna for mobile radio," in Proceedings of the 3rd Annual International Conference on Universal Personal Communications (ICUPC '94), pp. 230-235, San Diego, Calif, USA, September-October 1994.

[3] A. J. Weiss and B. Friedlander, "Maximum likelihood signal estimation for polarization sensitive arrays," IEEE Transactions on Antennas and Propagation, vol. 41, no. 7, pp. 918-925, 1993.

[4] X. Zhenhai, W. Xuesong, X. Shunping, and Z. Zhuang, "Filtering performance of polarization sensitive array: completely polarized case," Acta Electronica Sinica, vol. 32, no. 8, pp. 1310-1313, 2004.

[5] A. Smilde, R. Bro, and P. Geladi, Multi-Way Analysis. Applications in the Chemical Sciences, John Wiley \& Sons, Chichester, UK, 2004.

[6] R. A. Harshman, "Foundations of the PARAFAC procedure: model and conditions for an 'explanatory' multi-mode factor analysis," UCLA Working Papers Phonetics, vol. 16, no. 1, pp. 1-84, 1970. 
[7] J. D. Carroll and J.-J. Chang, "Analysis of individual differences in multidimensional scaling via an n-way generalization of "Eckart-Young" decomposition," Psychometrika, vol. 35, no. 3, pp. 283-319, 1970.

[8] L. De Lathauwer, B. De Moor, and J. Vandewalle, "Computation of the canonical decomposition by means of a simultaneous generalized Schur decomposition," SIAM Journal on Matrix Analysis and Applications, vol. 26, no. 2, pp. 295-327, 2004.

[9] L. De Lathauwer, "A link between the canonical decomposition in multilinear algebra and simultaneous matrix diagonalization," SIAM Journal on Matrix Analysis and Applications, vol. 28, no. 3, pp. 642-666, 2006.

[10] J. B. Kruskal, “Three-way arrays: rank and uniqueness of trilinear decompositions, with application to arithmetic complexity and statistics," Linear Algebra and Its Applications, vol. 18, no. 2, pp. 95-138, 1977.

[11] N. D. Sidiropoulos, G. B. Giannakis, and R. Bro, "Blind PARAFAC receivers for DS-CDMA systems," IEEE Transactions on Signal Processing, vol. 48, no. 3, pp. 810-823, 2000.

[12] N. D. Sidiropoulos, R. Bro, and G. B. Giannakis, "Parallel factor analysis in sensor array processing," IEEE Transactions on Signal Processing, vol. 48, no. 8, pp. 2377-2388, 2000.

[13] Y. Rong, S. A. Vorobyov, A. B. Gershman, and N. D. Sidiropoulos, "Blind spatial signature estimation via time-varying user power loading and parallel factor analysis," IEEE Transactions on Signal Processing, vol. 53, no. 5, pp. 1697-1710, 2005.

[14] Y. Yu and A. P. Petropulu, "Parafac based blind estimation of MIMO systems with possibly more inputs than outputs," in Proceedings of IEEE International Conference on Acoustics, Speech, and Signal Processing (ICASSP '06), vol. 3, pp. 133-136, Toulouse, France, May 2006.

[15] K. N. Mokios, N. D. Sidiropoulos, and A. Potamianos, "Blind speech separation using parafac analysis and integer least squares," in Proceedings of IEEE International Conference on Acoustics, Speech, and Signal Processing (ICASSP '06), vol. 5, pp. 73-76, Toulouse, France, May 2006.

[16] X. Zhang and D. Xu, "Blind PARAFAC receiver for space-time block-coded CDMA system," in Proceedings of International Conference on Communications, Circuits and Systems, vol. 2, pp. 675-678, Guilin, Guangzi, China, June 2006.

[17] X. Zhang and D. Xu, "PARAFAC multiuser detection for SIMO-CDMA system," in Proceedings of International Conference on Communications, Circuits and Systems, vol. 2, pp. 744747, Guilin, Guangzi, China, June 2006.

[18] S. A. Vorobyov, Y. Rong, N. D. Sidiropoulos, and A. B. Gershman, "Robust iterative fitting of multilinear models," IEEE Transactions on Signal Processing, vol. 53, no. 8, part 1, pp. 2678-2689, 2005.

[19] G. Tomasi and R. Bro, "A comparison of algorithms for fitting the PARAFAC model," Computational Statistics \& Data Analysis, vol. 50, no. 7, pp. 1700-1734, 2006.

[20] N. D. Sidiropoulos and X. Liu, "Identifiability results for blind beamforming in incoherent multipath with small delay spread," IEEE Transactions on Signal Processing, vol. 49, no. 1, pp. 228-236, 2001.

[21] J. G. Proakis, Digital Communications, McGraw-Hill, New York, NY, USA, 3rd edition, 1995.

[22] S. E. Leurgans, R. T. Ross, and R. B. Abel, "A decomposition for three-way arrays," SIAM Journal on Matrix Analysis and Applications, vol. 14, no. 4, pp. 1064-1083, 1993.
[23] E. Sanchez and B. R. Kowalski, "Tensorial resolution: a direct trilinear decomposition," Journal of Chemometrics, vol. 4, no. 1, pp. 29-45, 1990.

[24] D. Gesbert, J. Sorelius, and A. Paulraj, "Blind multi-user MMSE detection of CDMA signals," in Proceedings of IEEE International Conference on Acoustics, Speech, and Signal Processing (ICASSP '98), vol. 6, pp. 3161-3164, Seattle, Wash, USA, May 1998.

[25] M. K. Tsatsanis and Z. Xu, "Performance analysis of minimum variance CDMA receivers," IEEE Transactions on Signal Processing, vol. 46, no. 11, pp. 3014-3022, 1998.

Xiaofei Zhang received the M.S. degree in electrical engineering from Wuhan University, Wuhan, China, in 2001. He received the Ph.D. degree in communication and information systems from Nanjing University of Aeronautics and Astronautics in 2005. From 2005 to 2007, he was a Lecturer in Electronic Engineering Department, Nanjing University of Aeronautics and Astronautics, Nanjing, China. His research is focused on array signal processing and communication signal processing.

Dazhuan Xu graduated from Nanjing Institute of Technology, Nanjing, China, in 1983. He received the M.S. and Ph.D. degrees in communication and information systems from Nanjing University of Aeronautics and Astronautics in 1986 and 2001, respectively. He is now a Full Professor in the College of Information Science and Technology, Nanjing University of Aeronautics and Astronautics, Nanjing, China. His research inter-

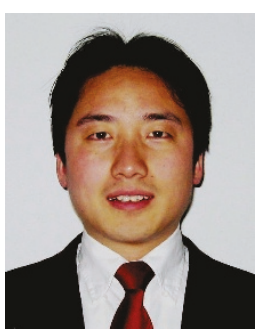
ests include digital communications, software radio, coding theory, and medical signal processing.

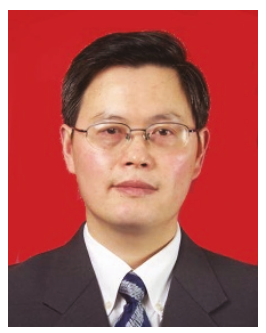

